\title{
Game Related Statistics Discriminate National and Foreign Players According to Playing Position and Team Ability in the Women's Basketball EuroLeague
}

\author{
Lorenzo Gasperi $^{1, *}$, Daniele Conte ${ }^{2} \mathbb{D}$, Anthony Leicht ${ }^{3}\left(\mathbb{D}\right.$ and Miguel-Ángel Gómez-Ruano ${ }^{1}(\mathbb{D}$ \\ 1 Facultad de Ciencias de la Actividad Física y del Deporte, Universidad Politécnica de Madrid, \\ 28031 Madrid, Spain; miguelangel.gomez.ruano@upm.es \\ 2 Institute of Sport Science and Innovations, Lithuanian Sports University, 44221 Kaunas, Lithuania; \\ daniele.conte@lsu.lt \\ 3 Sport and Exercise Science, James Cook University, Townsville 4814, Australia; anthony.leicht@jcu.edu.au \\ * Correspondence: lorenzo.gasperi@alumnos.upm.es; Tel.: +34-60-374-3700
}

Received: 19 June 2020; Accepted: 28 July 2020; Published: 30 July 2020

\begin{abstract}
The aim of the present study was to examine the differences in game-related statistics between national and foreign female basketball players in the Women's EuroLeague, according to playing positions and team ability. The official box-scores of 112 games from the 2016-2017 season of the Women's EuroLeague (FIBA) were examined. Players were categorised based upon country of nationality versus competition (i.e., foreign or national), playing positions (i.e., Guards, Forwards, Centers), and team ability (i.e., four groups using a cluster of k-means analysis according to the winning percentage of each team during the competition). A structural coefficient (SC) above $|0.30|$ was used to identify the variables that best differentiated the national and foreign players. Results showed that foreign players had a better performance according to team ability and playing position for most of the performance indicators, with higher values for minutes played, percentage of successful 2-point field-goals, percentage of successful free-throws, and percentage of assists. Moreover, foreign players performed better in variables associated with offensive situations, while national players were prevailing with indicators associated with defensive actions. These results have highlighted the unique contributions of foreign and national players, based upon playing position and team ability, to team success in the Euroleague. This information will assist the recruitment process of national and foreign athletes for coaches to develop successful elite female basketball teams.
\end{abstract}

Keywords: game-related statistics; team sports; discriminant scores; player position; team ability; foreign players; women basketball

\section{Introduction}

Basketball is a sport that encompasses interactions between physiological fitness, psychological preparedness, physical development, biomechanical proficiency, and tactical awareness, amongst several others [1]. Previous research investigating performance indicators in female basketball have provided a clear understanding of game performance requirements [2-4]. Defensive and offensive rebounds, successful 3-point and 2-point field-goals, assists, and inside game play tactics were reported to be the key performances indicators (KPIs) in female basketball as they increased the possibility of winning at national and international tournaments [5,6]. Further, situational variables such as team ability and player's position were identified as important factors for game performances [4,7-9]. For example, team ability, classified by the percentage of winning games during a basketball season $[8,10]$, was able to differentiate professional male teams in terms of game statistics (e.g., 2-point field-goals, passes, and defensive rebounds), with lower performances for teams with 
low team ability [8]. For playing position, Forwards were demonstrated to exhibit greater shooting efficacy inside the paint, which was more important to match outcome than that of other playing positions such as Guards and Centers [11]. However, these variables were mainly investigated in male basketball performances [4], while little information was available for female basketball players. Additionally, no previous study has assessed these variables during a female basketball competition, with further studies addressing the effect of team ability and playing position on basketball game-related statistics needed.

Understanding the performance profile of female basketball players in accordance with contextual factors is vital for teams to optimize recruitment of players (i.e., searching, scouting, and signing players). Female basketball players usually change teams, countries, and/or continents during their career based upon finding the team best suited to their playing characteristics and/or financial rewards [12]. Therefore, players will likely compete in their home nation as well as in foreign countries [13]. For example, the number of foreign players engaged with the National Basketball Association (NBA), which is the premier basketball league in the world, increased from 35 in 1999 to 110 in 2018 [14]. Similarly, the number of foreign players in the Women's NBA (WNBA) increased from 30 in the 2017/2018 season to 38 in the 2018/2019 season (information obtained from https://en.wikipedia.org/wiki/List_of_foreign_WNBA_players). This growth in foreign players may be driven by profit (e.g., expanding the team brand, followed by signing foreign top players) and/or performance by incorporating the most skilled players for greater team performance [14]. Therefore, the inclusion of both national and foreign players may affect team performance, with this impact unknown within female basketball competitions. Although, some evidence does exist for male basketball competitions, with the performance efficiency of foreign players across several seasons (from 1997 to 2010) within the Turkish Basketball League greater compared to native players [15]. Therefore, examination of female players' nationality in conjunction with contextual variables such as playing position and team ability may assist coaches to gain a better understanding of players' individual contribution to overall team performances [16]. Further, this information could assist coaches, managers, and performance analysts with effective decisions during recruitment processes. Therefore, the aim of the present study was to examine the differences in game-related statistics or performance between national and foreign basketball players in an elite female competition (EuroLeague), according to playing position and team ability.

\section{Materials and Methods}

\subsection{Data}

This study was a retrospective analysis of publically available data obtained from the 2016-2017 season, official box-scores of the FIBA Women's EuroLeague. Data obtained using this methodology across different contexts has exhibited good reliability [17,18]. All games from the initial group phase ( $\mathrm{n}=112$ games) were selected with the following game-related statistics gathered: 2 - and 3-point field-goal shots (both successful and unsuccessful), free-throw shots (both successful and unsuccessful), defensive and offensive rebounds, blocks, assists, fouls, steals, turnovers, and minutes played. These metrics have been examined routinely in prior studies of basketball player and team performances $[8,11]$.

Players were categorized as either national players (i.e., athletes playing for a club competing in the national competition of the nation of their own nationality) or foreign players (i.e., athletes playing for a club competing in a national competition that is different from the nation of their own nationality). For the 200 players within the 16 women's Basketball Euroleague teams, 119 were national players (59.5\% of the total) and 81 were foreign players (40.5\% of the total). For the 112 games examined in the current study that resulted in 2225 player observations, 1159 observations were from national players' (52.1\% of the total) and 1066 observations were from foreign players ( $47.9 \%$ of the total). Regarding Guards, 943 player observations included 528 from national players ( $56.0 \%$ of the total) and 415 were 
from foreign players ( $44.0 \%$ of the total). Regarding Forwards, 539 observations were from 287 national players (53.3\% of the total) and 252 were from foreign players ( $46.7 \%$ of the total). Regarding Centers, 743 observations were from 344 national players (46.3\% of the total) and 399 were from foreign players (53.7\% of the total) [8]. Moreover, team ability was determined by dividing the sample into four groups using a k-means cluster analysis according to the winning percentage of each team during the competition [winning $\%=$ (games won/total games) $* 100$ ]. The four groups based upon team ability were poor $(11.2 \pm 3.4 \%)$, low $(42.9 \pm 6.5 \%)$, medium $(65.5 \pm 6.6 \%)$, and high $(93.7 \pm 6.0 \%)$.

\subsection{Statistical Analyses}

Firstly, normality assumptions were checked for each group of players in each condition (i.e., national vs. foreign) using the Kolmogorov-Smirnov test. Secondly, a linear regression model was used to calculate the player's contribution to the team's performance for each game-related statistic as follows:

$$
\text { Variable } x=\beta 0+\beta 1 * \text { Player's performance variable } x+\varepsilon i \text {, }
$$

where $\beta 0$ is the intercept, $\beta 1$ is the impact of each predictor independent variable, and $\varepsilon i$ is the disturbance term.

Subsequently, the predictor model was used to calculate the individual players' performance for each variable with the constant $(\beta 0)$ divided by 5 to account for the number of players on court at one time (i.e., 5) in each model [19].

Thirdly, player's contribution to the team was then examined via discriminant analysis to identify the game-related statistics that best discriminated between national and foreign players according to team ability. The structural coefficient (SC) above $|0.30|$ identified the variables that best contributed to the differentiation of national and foreign players. Validation of each analysis was conducted using the leave-one-out classification models process [20]. All statistical analyses were conducted using PASW statistics 20 software (SPSS Inc., Chicago, IL, USA). The level of statistical significance was set at $p<0.05$.

\section{Results}

The means and standard deviations with univariate differences between national and foreign players for each game-related statistic per playing position and team ability are presented in Tables 1-3. The univariate analysis showed that foreign players exhibited greater values for most game-related statistics compared to national players based upon Guard, Forward, and Center playing positions (see Tables 1-3).

Table 1. Descriptive results (M, mean; SD, standard deviation) of national and foreign Guards based upon poor, low, medium, and high team ability.

\begin{tabular}{|c|c|c|c|c|c|c|c|c|c|c|}
\hline \multirow{4}{*}{ Variables } & \multicolumn{10}{|c|}{ Team Ability } \\
\hline & \multicolumn{5}{|c|}{ Poor } & \multicolumn{5}{|c|}{ Low } \\
\hline & \multicolumn{2}{|c|}{ National } & \multicolumn{2}{|c|}{ Foreign } & \multirow{2}{*}{ Sig. } & \multicolumn{2}{|c|}{ National } & \multicolumn{2}{|c|}{ Foreign } & \multirow{2}{*}{ Sig. } \\
\hline & $\mathbf{M}$ & SD & $\mathbf{M}$ & SD & & $\mathbf{M}$ & SD & $\mathbf{M}$ & SD & \\
\hline Minutes & 63082 & 35935 & 108453 & 26853 & $<0.01$ & 75479 & 38092 & 95881 & 38527 & $<0.01$ \\
\hline$\%$ Successful 2 pt & 9.24 & 2.37 & 12.94 & 3.38 & $<0.01$ & 10.42 & 2.94 & 11.37 & 3.53 & $<0.01$ \\
\hline$\%$ Unsuccessful 2 pt & 17.10 & 1.63 & 14.24 & 2.39 & $<0.01$ & 16.57 & 1.69 & 15.79 & 2.29 & $<0.01$ \\
\hline$\%$ Successful $3 \mathrm{pt}$ & 12.10 & 2.19 & 12.86 & 2.95 & 0.04 & 12.62 & 2.71 & 14.18 & 3.74 & $<0.01$ \\
\hline$\%$ Unsuccessful 3 pt & 14.03 & 0.26 & 14.02 & 0.30 & 0.70 & 14.08 & 0.24 & 13.87 & 0.33 & $<0.01$ \\
\hline$\%$ Successful free throws & 12.12 & 0.75 & 13.78 & 2.03 & $<0.01$ & 12.53 & 1.14 & 13.43 & 1.88 & $<0.01$ \\
\hline$\%$ Unsuccessful free throws & 13.87 & 0.04 & 13.89 & 0.07 & $<0.01$ & 13.88 & 0.05 & 13.89 & 0.05 & 0.17 \\
\hline$\%$ Offensive rebounds & 13.24 & 0.29 & 13.40 & 0.38 & $<0.01$ & 13.29 & 0.31 & 13.34 & 0.35 & 0.15 \\
\hline$\%$ Assists & 8.67 & 1.62 & 9.74 & 1.84 & $<0.01$ & 9.02 & 1.46 & 9.79 & 1.89 & 0.00 \\
\hline$\%$ Steals & 13.07 & 0.80 & 13.76 & 1.26 & $<0.01$ & 13.31 & 1.13 & 13.61 & 1.11 & 0.01 \\
\hline$\%$ Defensive rebounds & 13.38 & 0.56 & 12.92 & 0.72 & $<0.01$ & 13.30 & 0.63 & 12.96 & 0.72 & $<0.01$ \\
\hline$\%$ Fouls & 15.04 & 1.37 & 15.82 & 1.40 & $<0.01$ & 15.06 & 1.30 & 15.16 & 1.28 & 0.44 \\
\hline$\%$ Turnover & 14.49 & 1.03 & 15.04 & 1.11 & $<0.01$ & 14.53 & 0.92 & 14.97 & 1.23 & $<0.01$ \\
\hline$\%$ Blocks & 13.71 & 0.41 & 13.54 & 0.61 & 0.02 & 13.69 & 0.51 & 13.61 & 0.58 & 0.13 \\
\hline
\end{tabular}


Table 1. Cont.

\begin{tabular}{|c|c|c|c|c|c|c|c|c|c|c|}
\hline \multirow{3}{*}{ Variables } & \multicolumn{5}{|c|}{ Medium } & \multicolumn{5}{|c|}{ High } \\
\hline & \multicolumn{2}{|c|}{ National } & \multicolumn{2}{|c|}{ Foreign } & \multirow{2}{*}{ Sig. } & \multicolumn{2}{|c|}{ National } & \multicolumn{2}{|c|}{ Foreign } & \multirow{2}{*}{ Sig. } \\
\hline & $\mathbf{M}$ & SD & $\mathbf{M}$ & SD & & $\mathbf{M}$ & SD & $\mathbf{M}$ & SD & \\
\hline Minutes & 67214 & 29659 & 108157 & 22683 & $<0.01$ & 67406 & 36273 & 73706 & 22327 & 0.22 \\
\hline$\%$ Successful 2 pt & 9.68 & 2.39 & 11.79 & 4.41 & $<0.01$ & 11.08 & 3.42 & 10.63 & 2.55 & 0.39 \\
\hline$\%$ Unsuccessful 2 pt & 16.81 & 1.41 & 15.71 & 1.75 & $<0.01$ & 16.68 & 1.59 & 16.84 & 1.16 & 0.51 \\
\hline$\%$ Successful 3 pt & 12.39 & 2.27 & 13.60 & 3.05 & $<0.01$ & 12.75 & 2.86 & 14.35 & 4.73 & 0.04 \\
\hline$\%$ Unsuccessful 3 pt & 14.11 & 0.19 & 13.94 & 0.24 & $<0.01$ & 14.06 & 0.24 & 14.05 & 0.32 & 0.93 \\
\hline$\%$ Successful free throws & 12.54 & 1.20 & 13.50 & 1.95 & $<0.01$ & 12.53 & 1.16 & 13.17 & 1.74 & 0.03 \\
\hline$\%$ Unsuccessful free throws & 13.87 & 0.04 & 13.90 & 0.06 & $<0.01$ & 13.87 & 0.03 & 13.88 & 0.04 & 0.47 \\
\hline$\%$ Offensive rebounds & 13.30 & 0.29 & 13.37 & 0.35 & 0.11 & 13.20 & 0.17 & 13.27 & 0.29 & 0.13 \\
\hline$\%$ Assists & 9.02 & 1.72 & 10.48 & 2.13 & $<0.01$ & 8.84 & 1.23 & 9.41 & 1.38 & 0.02 \\
\hline$\%$ Steals & 13.46 & 1.06 & 13.72 & 0.96 & 0.09 & 13.26 & 0.88 & 13.36 & 0.96 & 0.58 \\
\hline$\%$ Defensive rebounds & 13.34 & 0.57 & 12.90 & 0.71 & $<0.01$ & 13.55 & 0.43 & 13.14 & 0.76 & $<0.01$ \\
\hline$\%$ Fouls & 15.04 & 1.24 & 15.63 & 1.26 & $<0.01$ & 14.46 & 1.14 & 14.81 & 1.11 & 0.08 \\
\hline$\%$ Turnover & 14.45 & 0.78 & 15.14 & 1.11 & $<0.01$ & 14.14 & 0.62 & 14.38 & 0.82 & 0.09 \\
\hline \% Blocks & 13.76 & 0.31 & 13.64 & 0.51 & 0.04 & 13.65 & 0.60 & 13.68 & 0.52 & 0.81 \\
\hline
\end{tabular}

Table 2. Descriptive results (M, mean; SD, standard deviation) of national and foreign Forwards based upon poor, low, medium, and high team ability.

\begin{tabular}{|c|c|c|c|c|c|c|c|c|c|c|}
\hline \multirow{4}{*}{ Variables } & \multicolumn{10}{|c|}{ Team Ability } \\
\hline & \multicolumn{5}{|c|}{ Poor } & \multicolumn{5}{|c|}{ Low } \\
\hline & \multicolumn{2}{|c|}{ National } & \multicolumn{2}{|c|}{ Foreign } & \multirow{2}{*}{ Sig. } & \multicolumn{2}{|c|}{ National } & \multicolumn{2}{|c|}{ Foreign } & \multirow{2}{*}{ Sig. } \\
\hline & $\mathbf{M}$ & SD & $\mathbf{M}$ & SD & & $\mathbf{M}$ & SD & $\mathbf{M}$ & SD & \\
\hline Minutes & 54963 & 23559 & 105630 & 13713 & $<0.01$ & 70839 & 40331 & 93701 & 36665 & $<0.01$ \\
\hline$\%$ Successful 2 pt & 8.91 & 1.61 & 12.77 & 3.45 & $<0.01$ & 10.38 & 3.64 & 13.15 & 4.37 & $<0.01$ \\
\hline$\%$ Unsuccessful 2 pt & 17.05 & 1.40 & 14.34 & 2.06 & $<0.01$ & 16.79 & 1.85 & 15.62 & 1.96 & $<0.01$ \\
\hline$\%$ Successful 3 pt & 11.37 & 1.78 & 12.70 & 2.63 & 0.01 & 12.84 & 2.88 & 12.80 & 3.10 & 0.93 \\
\hline$\%$ Unsuccessful 3 pt & 14.17 & 0.17 & 14.08 & 0.27 & 0.08 & 14.00 & 0.37 & 14.02 & 0.34 & 0.80 \\
\hline$\%$ Successful free throws & 12.31 & 1.06 & 13.11 & 1.76 & 0.02 & 12.73 & 1.78 & 13.19 & 1.54 & 0.04 \\
\hline$\%$ Unsuccessful free throws & 13.87 & 0.02 & 13.88 & 0.04 & 0.13 & 13.89 & 0.06 & 13.88 & 0.05 & 0.59 \\
\hline$\%$ Offensive rebounds & 13.25 & 0.27 & 13.69 & 0.66 & $<0.01$ & 13.41 & 0.43 & 13.66 & 0.54 & $<0.01$ \\
\hline$\%$ Assists & 7.99 & 0.79 & 8.44 & 1.21 & 0.07 & 8.20 & 1.14 & 8.72 & 1.17 & $<0.01$ \\
\hline$\%$ Steals & 12.76 & 0.41 & 13.45 & 0.67 & $<0.01$ & 13.37 & 1.09 & 13.57 & 1.25 & 0.21 \\
\hline$\%$ Defensive rebounds & 13.43 & 0.43 & 12.43 & 0.90 & $<0.01$ & 13.08 & 0.92 & 12.94 & 0.82 & 0.24 \\
\hline$\%$ Fouls & 15.43 & 1.31 & 16.09 & 1.24 & 0.04 & 14.86 & 1.24 & 15.45 & 1.33 & $<0.01$ \\
\hline$\%$ Turnover & 14.21 & 0.68 & 14.77 & 0.94 & 0.01 & 14.28 & 0.82 & 14.73 & 1.02 & $<0.01$ \\
\hline$\%$ Blocks & 13.67 & 0.78 & 13.31 & 0.76 & 0.06 & 13.56 & 0.63 & 13.37 & 0.87 & 0.07 \\
\hline \multirow{3}{*}{ Variables } & \multicolumn{5}{|c|}{ Medium } & \multicolumn{5}{|c|}{ High } \\
\hline & \multicolumn{2}{|c|}{ National } & \multicolumn{2}{|c|}{ Foreign } & \multirow{2}{*}{ Sig. } & \multicolumn{2}{|c|}{ National } & \multicolumn{2}{|c|}{ Foreign } & \multirow{2}{*}{ Sig. } \\
\hline & $\mathbf{M}$ & SD & $\mathbf{M}$ & SD & & $\mathbf{M}$ & SD & $\mathbf{M}$ & SD & \\
\hline Minutes & 33258 & 18751 & 96918 & 33552 & $<0.01$ & 31552 & 23689 & 111269 & 19188 & $<0.01$ \\
\hline$\%$ Successful 2 pt & 8.37 & 1.53 & 13.07 & 4.06 & $<0.01$ & 8.51 & 1.75 & 15.33 & 4.93 & $<0.01$ \\
\hline$\%$ Unsuccessful 2 pt & 17.57 & 1.04 & 15.28 & 2.20 & $<0.01$ & 17.98 & 0.88 & 14.20 & 1.99 & $<0.01$ \\
\hline$\%$ Successful 3 pt & 12.21 & 2.30 & 13.26 & 3.55 & $<0.01$ & 11.89 & 2.14 & 13.38 & 2.79 & $<0.01$ \\
\hline$\%$ Unsuccessful 3 pt & 14.18 & 0.19 & 14.06 & 0.29 & $<0.01$ & 14.19 & 0.16 & 14.07 & 0.20 & $<0.01$ \\
\hline$\%$ Successful free throws & 12.37 & 1.07 & 13.58 & 2.01 & $<0.01$ & 12.27 & 0.84 & 14.13 & 1.99 & $<0.01$ \\
\hline$\%$ Unsuccessful free throws & 13.87 & 0.03 & 13.90 & 0.06 & $<0.01$ & 13.87 & 0.03 & 13.91 & 0.05 & $<0.01$ \\
\hline$\%$ Offensive rebounds & 13.27 & 0.30 & 13.78 & 0.65 & $<0.01$ & 13.30 & 0.33 & 13.69 & 0.72 & $<0.01$ \\
\hline$\%$ Assists & 7.74 & 0.62 & 8.49 & 1.05 & $<0.01$ & 7.78 & 0.70 & 10.15 & 1.63 & $<0.01$ \\
\hline$\%$ Steals & 12.78 & 0.44 & 13.47 & 0.96 & $<0.01$ & 12.79 & 0.57 & 13.93 & 1.27 & $<0.01$ \\
\hline$\%$ Defensive rebounds & 13.56 & 0.43 & 12.38 & 1.10 & $<0.01$ & 13.48 & 0.48 & 11.87 & 1.11 & $<0.01$ \\
\hline$\%$ Fouls & 14.47 & 1.18 & 15.26 & 1.22 & $<0.01$ & 14.14 & 1.07 & 15.38 & 1.08 & $<0.01$ \\
\hline$\%$ Turnover & 13.87 & 0.53 & 14.69 & 1.02 & $<0.01$ & 13.81 & 0.37 & 14.86 & 0.96 & $<0.01$ \\
\hline$\%$ Blocks & 13.70 & 0.43 & 12.74 & 1.75 & $<0.01$ & 13.71 & 0.42 & 13.13 & 1.32 & $<0.01$ \\
\hline
\end{tabular}


Table 3. Descriptive results (M, mean; SD, standard deviation) of national and foreign Centers based upon poor, low, medium, and high team ability.

\begin{tabular}{|c|c|c|c|c|c|c|c|c|c|c|}
\hline \multirow{4}{*}{ Variables } & \multicolumn{10}{|c|}{ Team Ability } \\
\hline & \multicolumn{5}{|c|}{ Poor } & \multicolumn{5}{|c|}{ Low } \\
\hline & \multicolumn{2}{|c|}{ National } & \multicolumn{2}{|c|}{ Foreign } & \multirow{2}{*}{ Sig. } & \multicolumn{2}{|c|}{ National } & \multicolumn{2}{|c|}{ Foreign } & \multirow{2}{*}{ Sig. } \\
\hline & $\mathbf{M}$ & SD & $\mathbf{M}$ & SD & & $\mathbf{M}$ & SD & $\mathbf{M}$ & SD & \\
\hline Minutes & 23093 & 20769 & 99350 & 25994 & $<0.01$ & 71115 & 34005 & 92214 & 29669 & $<0.01$ \\
\hline$\%$ Successful 2 pt & 8.06 & 1.18 & 14.78 & 4.22 & $<0.01$ & 11.37 & 3.73 & 14.82 & 4.71 & $<0.01$ \\
\hline$\%$ Unsuccessful 2 pt & 17.78 & 1.10 & 14.78 & 2.06 & $<0.01$ & 15.84 & 1.93 & 14.60 & 2.12 & $<0.01$ \\
\hline$\%$ Successful 3 pt & 10.82 & 0.49 & 11.74 & 2.33 & 0.02 & 11.66 & 2.11 & 11.65 & 2.26 & 0.94 \\
\hline$\%$ Unsuccessful 3 pt & 14.30 & 0.04 & 14.14 & 0.28 & $<0.01$ & 14.19 & 0.17 & 14.20 & 0.20 & 0.64 \\
\hline$\%$ Successful free throws & 11.98 & 0.55 & 14.03 & 2.19 & $<0.01$ & 12.77 & 1.41 & 13.27 & 1.83 & $<0.01$ \\
\hline$\%$ Unsuccessful free throws & 13.87 & 0.04 & 13.89 & 0.09 & 0.22 & 13.89 & 0.06 & 13.90 & 0.07 & 0.03 \\
\hline$\%$ Offensive rebounds & 13.21 & 0.22 & 13.81 & 0.64 & $<0.01$ & 13.51 & 0.47 & 13.80 & 0.77 & $<0.01$ \\
\hline$\%$ Assists & 7.47 & 0.31 & 8.31 & 0.95 & $<0.01$ & 8.41 & 1.10 & 8.28 & 0.91 & 0.23 \\
\hline$\%$ Steals & 12.77 & 0.47 & 13.15 & 0.79 & 0.01 & 13.32 & 0.99 & 13.28 & 0.97 & 0.69 \\
\hline$\%$ Defensive rebounds & 13.70 & 0.43 & 12.17 & 1.17 & $<0.01$ & 12.83 & 0.86 & 12.32 & 1.11 & $<0.01$ \\
\hline$\%$ Fouls & 14.65 & 1.33 & 15.43 & 1.15 & $<0.01$ & 15.46 & 1.41 & 15.65 & 1.30 & 0.19 \\
\hline$\%$ Turnover & 13.88 & 0.56 & 14.71 & 0.87 & $<0.01$ & 14.52 & 0.89 & 14.62 & 0.92 & 0.28 \\
\hline$\%$ Blocks & 13.73 & 0.37 & 12.79 & 1.57 & $<0.01$ & 13.16 & 1.24 & 13.23 & 1.03 & 0.58 \\
\hline \multirow{3}{*}{ Variables } & \multicolumn{5}{|c|}{ Medium } & \multicolumn{5}{|c|}{ High } \\
\hline & \multicolumn{2}{|c|}{ National } & \multicolumn{2}{|c|}{ Foreign } & \multirow{2}{*}{ Sig. } & \multicolumn{2}{|c|}{ National } & \multicolumn{2}{|c|}{ Foreign } & \multirow{2}{*}{ Sig. } \\
\hline & M & SD & $\mathbf{M}$ & SD & & M & SD & M & SD & \\
\hline Minutes & 51562 & 28634 & 97377 & 20977 & $<0.01$ & 55396 & 24043 & 79901 & 21390 & $<0.01$ \\
\hline$\%$ Successful 2 pt & 11.05 & 3.87 & 16.23 & 4.47 & $<0.01$ & 12.10 & 4.30 & 16.08 & 4.80 & $<0.01$ \\
\hline$\%$ Unsuccessful 2 pt & 16.64 & 1.39 & 14.52 & 2.18 & $<0.01$ & 16.02 & 1.98 & 15.45 & 1.89 & 0.16 \\
\hline$\%$ Successful $3 \mathrm{pt}$ & 11.74 & 2.45 & 11.47 & 1.96 & 0.47 & 10.73 & 0.00 & 11.16 & 1.34 & 0.04 \\
\hline$\%$ Unsuccessful 3 pt & 14.19 & 0.22 & 14.18 & 0.22 & 0.87 & 14.30 & 0.03 & 14.27 & 0.08 & 0.01 \\
\hline$\%$ Successful free throws & 12.54 & 1.04 & 13.30 & 1.68 & $<0.01$ & 12.69 & 1.16 & 13.58 & 1.92 & 0.01 \\
\hline$\%$ Unsuccessful free throws & 13.88 & 0.06 & 13.92 & 0.08 & 0.01 & 13.90 & 0.07 & 13.89 & 0.05 & 0.54 \\
\hline$\%$ Offensive rebounds & 13.37 & 0.32 & 13.96 & 0.74 & $<0.01$ & 13.85 & 0.67 & 13.83 & 0.56 & 0.86 \\
\hline \% Assists & 7.73 & 0.60 & 8.66 & 1.10 & $<0.01$ & 8.08 & 0.88 & 8.32 & 0.84 & 0.18 \\
\hline$\%$ Steals & 12.84 & 0.47 & 13.35 & 0.91 & $<0.01$ & 13.06 & 0.65 & 13.44 & 1.01 & 0.04 \\
\hline$\%$ Defensive rebounds & 13.28 & 0.72 & 12.14 & 0.99 & $<0.01$ & 12.84 & 0.90 & 12.64 & 0.79 & 0.25 \\
\hline$\%$ Fouls & 14.87 & 1.35 & 15.11 & 1.31 & 0.28 & 15.29 & 1.47 & 15.30 & 1.39 & 0.98 \\
\hline$\%$ Turnover & 14.05 & 0.63 & 14.66 & 0.82 & $<0.01$ & 14.03 & 0.55 & 14.17 & 0.76 & 0.31 \\
\hline$\%$ Blocks & 13.22 & 1.24 & 12.81 & 1.43 & 0.08 & 12.66 & 1.60 & 11.58 & 2.19 & 0.01 \\
\hline
\end{tabular}

The discriminant functions that differentiated national and foreign players were all statistically significant for each team ability group $(p \leq 0.05)$, with the structural coefficients for each playing position and team ability displayed in Table 4 . The classification results for all functions were good to very good, with values greater than $69 \%$ for correct classification of cases [20].

When considering Guards, foreign players were discriminated from national players by $\%$ successful free-throws, \% defensive rebounds, and \% assists for all team ability clusters (i.e., poor, low, medium, and high, see Table 4). Specifically, foreign Guards exhibited higher values for $\%$ successful free-throws and \% assists, while lower values for \% defensive rebounds compared to national Guards (see Table 1). In addition, the variables \% unsuccessful 2-point field-goals (lower values for foreign players, see Table 1) and minutes played (higher values for foreign players, see Table 1) discriminated foreign and national Guards for all team ability clusters, except for the high level cluster (see Table 4).

When considering Forwards, foreign players were discriminated from national players by $\%$ minutes played and \% successful and unsuccessful 2-point field-goals for all team ability clusters (see Table 4). Foreign players exhibited higher values for minutes played and \% successful 2-point field-goals and lower values for \% unsuccessful 2-point field-goals compared to national players (see Table 2). Additionally, foreign Forwards exhibited statistically higher values for $\%$ assists and $\%$ turnovers compared to national forwards (see Table 2) when discriminated in low, medium, and high team ability clusters (see Table 4 ). 
Table 4. Discriminant function structure coefficients (SC) and tests of significance for each game metric that differentiated foreign and national Guards, Forwards, and Centers, based upon poor, low, medium, and high team ability.

\begin{tabular}{|c|c|c|c|c|c|c|c|c|c|c|c|c|}
\hline \multirow{3}{*}{ Variables } & \multicolumn{4}{|c|}{ Guards } & \multicolumn{4}{|c|}{ Forwards } & \multicolumn{4}{|c|}{ Centers } \\
\hline & Poor & Low & Med & High & Poor & Low & Med & High & Poor & Low & Med & High \\
\hline & SC & SC & SC & SC & SC & SC & SC & SC & SC & SC & SC & SC \\
\hline Minutes & 0.68 & -0.52 & 0.84 & 0.21 & 0.70 & 0.55 & 0.91 & 0.72 & 0.90 & -0.59 & 0.80 & 0.66 \\
\hline$\%$ Successful 2 pt & 0.64 & -0.29 & 0.34 & -0.14 & 0.43 & 0.64 & 0.59 & 0.40 & 0.54 & -0.74 & 0.51 & 0.53 \\
\hline$\%$ Unsuccessful 2 pt & -0.71 & 0.39 & -0.39 & 0.11 & -0.45 & -0.57 & -0.52 & -0.53 & -0.48 & 0.56 & -0.47 & -0.18 \\
\hline$\%$ Successful 3 pt & 0.15 & -0.49 & 0.25 & 0.35 & 0.17 & -0.01 & 0.14 & 0.12 & 0.14 & 0.01 & -0.05 & 0.27 \\
\hline$\%$ Unsuccessful 3 pt & -0.03 & 0.75 & -0.45 & -0.02 & -0.12 & 0.03 & -0.20 & -0.13 & -0.19 & -0.04 & -0.01 & -0.36 \\
\hline$\%$ Successful free throws & 0.57 & -0.60 & 0.34 & 0.37 & 0.16 & 0.26 & 0.29 & 0.26 & 0.32 & -0.28 & 0.22 & 0.34 \\
\hline$\%$ Unsuccessful free throws & 0.21 & -0.13 & 0.28 & 0.12 & 0.10 & -0.07 & 0.22 & 0.18 & 0.07 & -0.20 & 0.19 & -0.08 \\
\hline$\%$ Offensive rebounds & 0.24 & -0.14 & 0.13 & 0.25 & 0.26 & 0.47 & 0.39 & 0.15 & 0.32 & -0.42 & 0.40 & -0.02 \\
\hline$\%$ Assists & 0.30 & -0.46 & 0.42 & 0.39 & 0.13 & 0.42 & 0.34 & 0.41 & 0.30 & 0.11 & 0.41 & 0.17 \\
\hline$\%$ Steals & 0.33 & -0.27 & 0.14 & 0.09 & 0.36 & 0.16 & 0.36 & 0.25 & 0.16 & 0.04 & 0.28 & 0.27 \\
\hline$\%$ Defensive rebounds & -0.36 & 0.49 & -0.38 & -0.56 & -0.42 & -0.15 & -0.55 & -0.40 & -0.44 & 0.46 & -0.54 & -0.15 \\
\hline$\%$ Fouls & 0.28 & -0.08 & 0.26 & 0.29 & 0.14 & 0.43 & 0.26 & 0.23 & 0.19 & -0.12 & 0.08 & 0.00 \\
\hline$\%$ Turnover & 0.26 & -0.41 & 0.40 & 0.29 & 0.20 & 0.45 & 0.39 & 0.31 & 0.30 & -0.10 & 0.34 & 0.13 \\
\hline$\%$ Blocks & -0.17 & 0.15 & -0.17 & 0.04 & -0.13 & -0.23 & -0.29 & -0.13 & -0.21 & -0.05 & -0.13 & -0.35 \\
\hline Eigenvalue & 1.020 & 0.248 & 0.823 & 0.278 & 3.211 & 0.296 & 1.658 & 6.177 & 2.625 & 0.307 & 1.342 & 0.678 \\
\hline$\%$ of Variance & 100 & 100 & 100 & 100 & 100 & 100 & 100 & 100 & 100 & 100 & 100 & 100 \\
\hline Canonical Correlation & 0.711 & 0.446 & 0.672 & 0.467 & 0.873 & 0.478 & 0.79 & 0.928 & 0.851 & 0.484 & 0.757 & 0.636 \\
\hline Wilks' Lambda & 0.495 & 0.801 & 0.548 & 0.782 & 0.237 & 0.772 & 0.376 & 0.139 & 0.276 & 0.765 & 0.427 & 0.596 \\
\hline Chi-square & 129.409 & 92.869 & 108.705 & 30.215 & 86.262 & 54.932 & 122.18 & 206.948 & 133.941 & 101.884 & 116.588 & 43.455 \\
\hline $\mathrm{df}$ & 14 & 14 & 14 & 14 & 14 & 14 & 14 & 14 & 14 & 14 & 14 & 14 \\
\hline Sig. & $<0.001$ & $<0.001$ & $<0.001$ & 0.007 & $<0.001$ & $<0.001$ & $<0.001$ & $<0.001$ & $<0.001$ & $<0.001$ & $<0.001$ & $<0.001$ \\
\hline Classification results $\%$ & 83.9 & 70.0 & 81.1 & 69.7 & 100 & 73.8 & 91.0 & 99.1 & 92.0 & 70.8 & 84.9 & 80.6 \\
\hline
\end{tabular}


For the Center playing position, foreign players were discriminated from national Centers by $\%$ minutes played and \% successful 2-point field-goals for all investigated team ability clusters (see Table 4), with greater values for foreign players (see Table 3). Furthermore, foreign Centers exhibited lower values of $\%$ unsuccessful 2-point field-goals and \% defensive rebounds compared to national Centers (see Table 3) when discriminated in poor, low, and medium team ability clusters (see Table 4). Conversely, in the same team ability clusters (see Table 4), foreign players exhibited higher values of $\%$ offensive rebounds (see Table 4).

\section{Discussion}

The purpose of the present study was to identify the game-related statistics best discriminating national and foreign female basketball players according to playing positions and team ability. From a general point of view, the results showed that foreign players have a better performance according to team ability and playing position for most of the performance indicators, with higher values specifically for $\%$ minutes played, \% successful 2-point field-goal, \% successful free-throws, and \% assists. These findings will be of extreme importance for coaches and managers when recruiting players based on their performance strengths and weaknesses, their playing position, and nationality $[14,15]$.

This investigation provided a better understanding about the game performance of foreign and national female basketball players. Specifically, foreign Guards had better performance in \% successful free-throws and \% assists compared to national players. These results reflect the importance of foreign players during collaborative actions and drawing fouls since assists and free-throws, respectively, are considered two of the main KPIs in female basketball success [21,22]. One interesting finding from our results was the questionable differences for 2- and 3-point field-goals for Guards, where there was no consistent evidence for all clustered groups, despite this offensive match metric being a prominent focus for the playing position [11]. This finding indicated that Guards with similar, and likely very good, field-goal shooting ability were recruited within the Euroleague, irrespective of nationality. Conversely, foreign Guards had lower \% defensive rebounds compared to national players, which is indicative of a greater offensive focus for foreign players compared to national players who were more defensive orientated. Subsequently, these greater skills (i.e., shooting, ball-handling, team-work, and assisting) should be primarily considered when recruiting foreign players to complement national players in the assembly of a successful team.

While the previously discussed variables differentiated national and foreign Guards regardless of team ability, the variable $\%$ minutes played discriminated between foreign and national players within the poor to medium clusters only. These findings indicated that lower level teams were more dependent on foreign Guards playing for success compared to national Guards. A possible explanation for this result might be due to the perceived regard of foreign players' ability by coaches and teams [15] and typically greater financial investment in such players [14]. Further, it may be a result of the poorer shooting percentage (\% unsuccessful 2-point field-goals) in national players and greater 3-point shooting by foreign players in these clusters (See Table 2), which are key statistics for success in female basketball $[3,23]$. Conversely, no differences were found in $\%$ minutes played between national and foreign Guards for the high cluster group. Possibly, high-level teams rely less on foreign Guards as a dominant role as teams recruit quality national Guards, which enables greater flexibility in this position for the team. Overall, these results highlight the importance of foreign Guards across all clusters, with greater reliance on these players to provide unique contributions to team success within lower team ability levels.

For the Forwards, a better 2-point shooting performance for foreign players compared to national players was identified for each team ability cluster, which matched with the higher \% unsuccessful 2-point field-goals for national compared to foreign players. These results again confirm the importance of foreign players to team success as 2-point shooting ability has been highlighted as a fundamental performance indicator that differentiates winning and losing female basketball teams [4,24]. Foreign players in this position also accumulated more playing time compared to national Forwards across 
all team ability clusters, further highlighting teams' reliance on these foreign players. This reliance was also reflected in the greater values of $\%$ assist and \% turnovers for foreign players within the low, medium, and high team ability clusters. Due to the higher number of minutes played by foreign players compared to national players, foreign Forwards were likely able to perform more effective actions prior to scoring (i.e., assists), which is another key performance indicator in basketball [5,25-28]. Additionally, foreign Forwards performed a higher \% of turnovers compared to national players, most likely due to their involvement in a greater number of ball possessions at the low-high team ability levels. Despite low turnovers being an important indicator that is able to discriminate between winning and losing elite female teams $[5,24]$, the current results may indicate a potential risky behaviour of foreign players that may impact team performance and success. Coaches should consider this possible risky behaviour when considering foreign Forwards during recruitment.

Similar to the other playing position results, higher values for $\%$ minutes played, $\%$ successful 2-point field-goals, and \% offensive rebounds were observed for foreign Centers (see Table 4). These results indicated a greater offensive ability for foreign Centres compared to national Centers. Further, national Centers exhibited poorer offensive (greater \% unsuccessful 2-point field goals) and greater defensive (\% defensive rebounds) actions, with these differences most notable at the lower team ability levels (poor-medium). National Centers also exhibited a greater defensive action (\% blocks) compared to foreign Centers within the high team ability cluster. These results indicate that foreign and national Centers had distinct and different primary roles within the Euroleague, despite all aligning with the main offensive and defensive actions reported for Centers [11,21]. This distinction further highlighted the unique foci and use of foreign players within the Euroleague Women's competition, with coaches and teams encouraged to consider this focus when developing team rosters. In fact, these results may reflect: (i) an improvement of more assertive and defensive behaviours for national players (higher commitment to their team) due to the recruitment of highly skilled foreign players for their offensive skills which national players compete/train against [29]; and (ii) the supplementary recruitment process of coaches and managers for national players to complement the skills and abilities of foreign players in this specific playing position [15].

To the best of our knowledge, this is the first study analysing the differences in game-related statistics between national and foreign elite female players according to different playing positions and team abilities. This novelty makes comparisons with other studies difficult, with some limitations needing discussion for future directions. Firstly, only games from the initial phase of the Euroleague Women's competition were analysed for this study. Future studies might provide additional insight about the differences in game-related statistics between national and foreign female basketball players during the final competition phase, where game metrics were reported to be important for match success and different to initial competition phases [4,5]. Secondly, only the top European female basketball league was investigated, providing results for a specific basketball population, therefore future investigations are encouraged to examine other top leagues worldwide such as the WNBA. Finally, this study only examined one year of game statistics and it would be interesting to see if these differences between national and foreign players are stable or evolve over time. Future studies of larger datasets across multiple years will confirm the enduring impact of foreign and national female players on team performance to assist coaches with effective recruitment processes.

\section{Conclusions}

The discriminatory power of game-related statistics allowed us to identify the unique performances that national and foreign players exhibit according to team ability and playing position. Specifically, the main results revealed that foreign players exhibited higher values for: (i) playing time; (ii) shooting performances for free-throws and 2-point field-goals for the majority of clustered playing positions; (iii) team work (i.e., assists) for Guard and Forward players; (iv) securing defensive (i.e., Guards) and offensive rebounds (Centers); and (v) turnovers for Forwards. These results provide more information for managers to consider when recruiting national and foreign athletes for successful, elite female 
basketball teams. Further, these results will help coaches to better prepare technical-tactical training sessions so that individualized work can resolve specific and important vulnerabilities for greater player performance during competition.

Author Contributions: Conceptualization, M.-Á.G.-R. and L.G.; Methodology, M.-Á.G.-R.; Software, M.-Á.G.-R.; Validation, M.-Á.G.-R., L.G., D.C., and A.L.; Formal analysis, M.-Á.G.-R.; Data Collection, L.G.; Writing-Original Draft Preparation, L.G., M.-Á.G.-R., and D.C.; writing-Review and Editing, D.C. and A.L.; Supervision, M.-Á.G.-R., D.C., and A.L.; Funding Acquisition, L.G. All authors have read and agreed to the published version of the manuscript.

Funding: This research received no external funding.

Acknowledgments: This research was supported by "Consejo Social" of Universidad Politécnica de Madrid, with the Project "XVII Convocatoria de Ayudas del Consejo Social para el Fomento de la Formación y la Internacionalización de Doctorandos para el curso 2018-2019".

This study was supported by a mobility Grant "Salvador de Madariaga" (PRX18/00098; Ministry of Education, Culture and Sport of Spain) for the author Miguel-Ángel Gómez during a research exchange at James Cook University (Townsville, Australia).

Conflicts of Interest: The authors declare no conflict of interest.

\section{References}

1. Glazier, P. Towards a grand unified theory of sports performance. Hum. Mov. Sci. 2017, 56, 139-156. [CrossRef] [PubMed]

2. Sampaio, J.; Godoy, S.I.; Feu, S. Discriminative power of basketball game-related statistics by level of competition and sex. Percept. Mot. Skills 2004, 99 (Suppl. 3), 1231-1238. [CrossRef] [PubMed]

3. Gómez, M.A.; Lorenzo, A.; Sampaio, J.; Ibáñez, S.J. Differences in game-related statistics between winning and losing teams in women's basketball. J. Hum. Mov. Stud. 2006, 51, 357-369.

4. Gómez, M.A.; Lorenzo, A.; Ibañez, S.J.; Sampaio, J. Ball possession effectiveness in men's and women's elite basketball according to situational variables in different game periods. J. Sports Sci. 2013, 31, 1578-1587. [CrossRef] [PubMed]

5. Leicht, A.S.; Gómez, M.A.; Woods, C.T. Team performance indicators explain outcome during women's basketball matches at the Olympic Games. Sports 2017, 5, 96. [CrossRef]

6. Şentuna, M.; Şentuna, N.; Ozdemir, N.; Serter, K.; Özen, G. The investigation of the effects of some variables in the playoff games played in Turkey women's basketball super league between 2013-2017 on winning and losing. Phys. Educ. Stud. 2018, 22, 146-150. [CrossRef]

7. Moreno, E.; Gómez, M.A.; Lago, C.; Sampaio, J. Effects of starting quarter score, game location, and quality of opposition in quarter score in elite women's basketball. Kinesiology 2013, 45, 48-54.

8. Sampaio, J.; Drinkwater, E.; Leite, N. Effects of season period, team quality, and playing time on basketball players' game-related statistics. Eur. J. Sport Sci. 2010, 10, 141-149. [CrossRef]

9. Ibáñez, S.J.; Sampaio, J.; Sáenz-López, P.; Giménez, J.; Janeira, M.A. Game statistics discriminating the final outcome of junior world basketball championship matches (Portugal 1999). J. Hum. Mov. Studies 2003, 45, $1-20$.

10. Pollard, R.; Pollard, G. Long-term trends in home advantage in professional team sports in North America and England (1876-2003). J. Sports Sci. 2005, 23, 337-350. [CrossRef]

11. Sampaio, J.; Janeira, M.A.; Ibáñez, S.J.; Lorenzo, A. Discriminant analysis of game-related statistics between basketball guards, forwards and centres in three professional leagues. Eur. J. Sport Sci. 2006, 6, 173-178. [CrossRef]

12. Hoffer, A.J.; Freidel, R. Does salary discrimination persist for foreign athletes in the NBA? Appl. Econ. Lett. 2014, 21, 1-5. [CrossRef]

13. Maderer, D.; Holtbrügge, D.; Schuster, T. Professional football squads as multicultural teams. Int. J. Cross Cult. Manag. 2014, 14, 215-238. [CrossRef]

14. Winkler, J. Examining the Impact of Foreign NBA Players on the Wages and Employment of American Basketball Players. Ph.D. Thesis, Department of Economics, Haverford College, Haverford, PA, USA, 2016. Available online: https://scholarship.tricolib.brynmawr.edu/handle/10066/18620 (accessed on 18 July 2020). 
15. Özmen, M.U. Foreign player quota, experience and efficiency of basketball players. J. Quant. Anal. Sports 2012, 8. [CrossRef]

16. Perez, S.J.; Siegler, M.V. The NBA and the influx of international basketball players. Appl. Econ. 2004, 36, 1009-1020. [CrossRef]

17. Sampaio, J.; Lorenzo, A.; Gómez, M.A.; Matalarranha, J.; Ibáñez, S.J.; Ortega, E. Analysis of discriminant statistics in basketball players according to their specific position, in the finals of European competitions (1988-2006). Differences between regular and substitute players. Apunt. Phys. Educ. Sports 2009, 2, 53-58.

18. Ibáñez, S.J.; González-Espinosa, S.; Feu, S.; García-Rubio, J. Basketball without borders? Similarities and differences between the Continental Basketball Championships. RICYDE Int. J. Sports Sci. 2018, 14, 42-54.

19. Severini, T.A. Analytic Methods in Sports: Using Mathematics and Statistics to Understand Data from Baseball, Football, Basketball, and Other Sports; Crc Press: Raton, Florida, USA, 2020.

20. Tabachnick, B.G.; Fidell, L.S. Experimental Designs Using ANOVA; Thomson/Brooks/Cole: Belmont, CA, USA, 2007; p. 724.

21. Sampaio, J.; Ibañez Godoy, S.J.; Gómez Ruano, M.Á.; Lorenzo Calvo, A.; Ortega Toro, E. Game location influences basketball players performance across playing positions. Int. J. Sport Psychol. 2008, 39, 43-50.

22. Teck, K.K.; Wang, J.; Mallett, C. Discriminating factors between successful and unsuccessful elite youth Olympic female basketball teams. Int. J. Perform. Anal. Sport 2012, 12, 119-131. [CrossRef]

23. Gòmez, M.-Á.; Lorenzo, A.; Ortega, E.; Sampaio, J.; Ibàñez, S.-J. Game related statistics discriminating between starters and nonstarters players in women's national basketball association league (WNBA). J. Sports Sci. Med. 2009, 8, 278-283.

24. Conte, D.; Lukonaitienè, I. Scoring strategies differentiating between winning and losing teams during FIBA EuroBasket women 2017. Sports 2018, 6, 50. [CrossRef] [PubMed]

25. Sampaio, J.; Janeira, M. Statistical analyses of basketball team performance: Understanding teams' wins and losses according to a different index of ball possessions. Int. J. Perform. Anal. Sport 2003, 3, 40-49. [CrossRef]

26. Csátaljay, G.; O’Donoghue, P.; Hughes, M.; Dancs, H. Performance indicators that distinguish winning and losing teams in basketball. Int. J. Perform. Anal. Sport 2009, 9, 60-66. [CrossRef]

27. Gómez, M.; Lorenzo, A.; Sampaio, J.; Ibañez, S.; Ortega, E. Game-related statistics that discriminated winning and losing teams from the Spanish men's professional basketball teams. Coll. Antropol. 2008, 32, 451-456.

28. Ibáñez, S.J.; García, J.; Feu, S.; Lorenzo, A.; Sampaio, J. Effects of consecutive basketball games on the game-related statistics that discriminate winner and losing teams. J. Sports Sci. Med. 2009, 8, 458-462. [PubMed]

29. Alvarez, J.; Forrest, D.; Sanz, I.; Tena, J.D. Impact of importing foreign talent on performance levels of local co-workers. Labour Econ. 2011, 18, 287-296. [CrossRef] 